\section{Allergie gegen Hasel- und Erdnüsse: Kreuzreaktion oder Zufall?}

Viele Patienten sind sowohl gegen Hasel- als auch gegen Erdnüsse als Nahrungsmittel allergisch, obwohl beide Pflanzen nicht miteinander verwandt sind. Gibt es bei diesen Früchten bislang unbekannte biologische Kreuzreaktionen oder ist die gemeinsame Sensibilisierung nur der atopischen Diathese oder dem Zufall geschuldet?
등 rdnüsse (Arachis hypogaea), gehören

Ezur Pflanzenfamilie der Legumino-

气ٌ̂ sen (Hülsenfrüchte), während die Hasel-

nuss (Corylus avellana) botanisch verwandt mit der Birke (Betula vulgaris) und der Erle ist, den Allergologen auch bekannt als „die drei Frühblüher“. Zur Frage, ob mögliche Allergene beider Pflanzen eventuell eine echte Kreuzreaktion oder biologisch ähnliche Proteinanteile aufweisen, untersuchten holländische Allergologen 81 Kinder und 80 Erwachsene mit einer Sensibilisierung gegen Haselnussallergene retrospektiv mittels ImmunoCAP.

Sie testeten mögliche Kreuzreaktionen durch Inhibitionsversuche bei 14 Probanden, die eine starke IgE-Reaktion gegen Erdnuss- oder Haselnussproteine (Ara h 1, 2, 3, 8, 9 bzw. Cor a 1, 8, 9, 14) aufwiesen. Außerdem wurde das Birkenallergen Bet v 1 untersucht. Die klinische Diagnostik beruhte auf der klas- sischen oralen Nahrungsmittelprovokation (doppelblind,placebokontrolliert) und/oder einer detaillierten Berücksichtigung von Anamnese und Symptomatik.

Die meisten Haselnuss-Sensibilisierten waren auch gegen das Birkenpollenallergen Bet v 1 sensibilisiert und zwar zu $80 \%$. Bei einer Sensibilisierung gegen Cor a 1 und Ara h 8 bestand ebenfalls eine Sensibilisierung gegen dieses Birkenpollenallergen.

Bei den Haselnuss-sensibilisierten Patienten konnten bei etwa zwei Drittel (68\%) zusätzliche Erdnusssensibilisierungen beobachtet werden, fast die Hälfte $(43 \%)$ zeigte außerdem eindeutige Symptome einer Erdnussallergie. Hier gab es aber keinerlei Assoziationen zum Bestehen oder der Ausprägung einer klinisch fassbaren Haselnussallergie.

Es lagen aber seltene Korrelationen einer IgE-Reaktivität mit Erdnussprotei- nen vor, bei zwei von 13 Patienten konnte man deutliche Kreuzreaktivitäten zwischen Ara h 3 und Cor a 9 nachweisen, sodass in seltenen Fällen durchaus die Sensibilisierung gegen Erdnuss eine Allergie gegen Haselnuss hervorrufen mag.

Masthoff LJ et al. Peanut allergy is common among hazelnut-sensitized subjects but is not primarily the result of IgE cross-reactivity. Allergy 2015;70:265-74

\section{Kommentar}

Eine Erdnussallergie ist im Allgemeinen nicht auf Kreuzreaktionen gegen Haselnussallergene zurückzuführen. Eine zusätzliche Erdnussallergie bei Haselnuss-sensibilisierten Patienten scheint damit in der Regel zufällig zu sein. Sehr selten kann eine primäre Ara-h-3-Sensibilisierung mögliche Ursache einer Haselnussallergie sein.

Insbesondere der Nachweis von spezifischem IgE gegen Cor a 14 respektive Ara h 2 sind aussagekräftige Marker für eine primäre Sensibilisierung gegen Haselnuss oder Erdnuss und lassen auch keine Kreuzreaktivität erkennen. Dr. Ulrich Mutschler

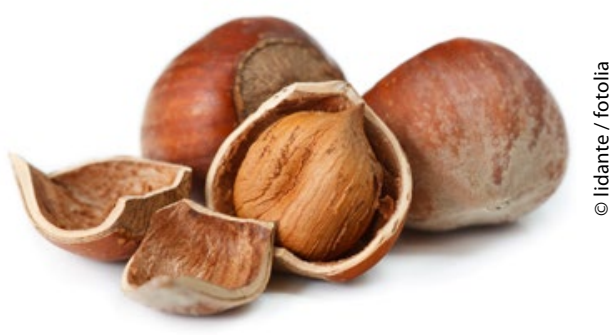

der von ADHS betroffenen Gruppe bis zum Alter von 6 Jahren signifikant seltener auf. Jedoch kam es ab dem Zeitpunkt der Diagnose bei den Jungen mit ADHS signifikant häufiger zu Übergewicht im Vergleich zur Kontrollgruppe. Die Körperlänge hingegen war geringer. Einflüsse von sozio-ökonomischen Status, Geburtsgewicht, Frühgeburtlichkeit und komorbiden Störungen sowie eine medikamentöse Therapie wurden dabei berücksichtigt.

Die Autoren vermuten, dass ein Grund für die Ergebnisse ein niedrigeres Selbstbewusstsein und soziale Isolation - beides häufiger bei Kindern mit ADHS zu finden - sind. Dadurch nehmen die Kinder weniger an sportlichen 